04

\title{
Разделение изотопов кальция в плазменной центрифуге с горячей стенкой
}

\author{
(C) В.Д. Борисевич ${ }^{1}$, Е.П. Потанин ${ }^{1,2}$ \\ ${ }^{1}$ Национальный исследовательский ядерный университет „МИФИ“, Москва, Россия \\ ${ }^{2}$ Национальный исследовательский центр „Курчатовский институт“, Москва, Россия \\ E-mail: VDBorisevich@mephi.ru
}

Поступило в Редакцию 6 февраля 2018 г.

Предлагается разделять изотопы кальция в противоточной плазменной центрифуге с горячей стенкой и отбором разделенных фракций путем осаждения паров на торцах устройства. Центробежное поле сил, обеспечивающее радиальный эффект разделения, возбуждается за счет ускорения слабоионизованной кальциевой плазмы вращающимся магнитным полем, а осевое циркуляционное течение, умножающее радиальный эффект разделения, создается бегущим магнитным полем. Возможности метода демонстрируются на примере расчета зависимости коэффициента разделения плазменной центрифуги от параметра, характеризующего интенсивность циркуляции.

DOI: 10.21883/PJTF.2019.01.47147.17242

Применение стабильных изотопов различных химических элементов часто оказывается невозможным из-за отсутствия подходящих методов их разделения. Наличие газообразного соединения, в состав которого входит разделяемая смесь изотопов, позволяет использовать традиционные газовые центрифуги только в том случае, если давление насыщенного пара составляет не менее 5-10 mm $\mathrm{Hg}$ [1]. Целый ряд элементов не имеет удобных газообразных соединений. Для разделения изотопов таких элементов в работе [2] была исследована возможность применения плазменной фазы. В [3] для создания центробежных сил в плазме было предложено использовать радиочастотные поля. Предпринимались также попытки создавать вращающуюся плазму в системе со стационарными скрещенными электрическим и магнитным полями при наличии электронного пучка [4].

Особый интерес представляет разделение изотопов кальция, нейтронно-избыточный изотоп которого $(\mathrm{Ca}-48)$ используется в ядерной физике для обеспечения исследований по получению новых трансурановых элементов [5], а также при определении природы массы нейтрино и ее абсолютной величины (Ca-40) [6]. Кальций имеет низкую упругость пара при комнатной температуре, вследствие чего его изотопы нельзя получать с помощью газовых центрифуг. Попытки использования лазерного метода для разделения изотопов кальция, проводившиеся с начала 70-х годов прошлого века, также не дали положительного результата [7].

В настоящее время разделение изотопов кальция производится в электромагнитных сепараторах. Малая производительность этого метода и связанная с этим высокая цена изотопа Са-48 мотивируют разработку альтернативных способов обогащения.

В работе [8] предложена концепция циркуляционной плазменной центрифуги с холодной боковой стенкой, в которой вращение слабоионизованной изотопной га- зовой смеси производится вращающимся магнитным полем, а необходимое для умножения радиального разделительного эффекта осевое циркуляционное течение создается за счет бегущей магнитной волны. Отметим, что в такой системе возможно разделение изотопов только химически неагрессивных элементов с высокой упругостью пара при нормальных условиях.

В основе предлагаемого принципиально нового решения проблемы разделения изотопов кальция лежит конструкция циркуляционной плазменной центрифуги с горячей боковой стенкой разделительной камеры (рис. 1). Предполагается, что вращение слабоионизованной плазмы создается за счет вращающегося с угловой скоростью поперечного магнитного поля, возбуждаемого осевыми токонесущими стержнями с соответствующими сдвигами фаз колебаний. Циркуляционный поток в зоне разделения возбуждается бегущим магнитным полем, создаваемым линией задержки. Радиальное магнитное поле в бегущей магнитной волне изменяется в пространстве и времени по закону $B_{r}=B_{1} \cos \left[\omega_{1}\left(t-z / V_{p h}\right)\right]$, где $\omega_{1}$ - круговая частота бегущей магнитной волны, $B_{1}-$ амплитудное значение магнитной индукции бегущего поля, $V_{p h}-$ фазовая скорость волны, $z-$ продольная координата.

Разделительный модуль помещается в водоохлаждаемую камеру 1, первоначально откачиваемую до давления порядка $10^{-5}$ Torr. Питание разделительной системы в паровой фазе осуществляется с помощью нагретой осевой трубки 2. Отбор обогащенного и обедненного продукта предполагается производить с помощью холодных металлических дисков 3 и 11, устанавливаемых в верхней и нижней частях колонны соответственно. Температура этих элементов должна быть такой, чтобы обеспечить осаждение паров кальция $\left(T_{\text {cool }} \approx 320-350 \mathrm{~K}\right)$. Напыление на них обогащенной смеси осуществляется через небольшие расположенные по азимуту отверстия 4,12 в торцевых горячих дис- 


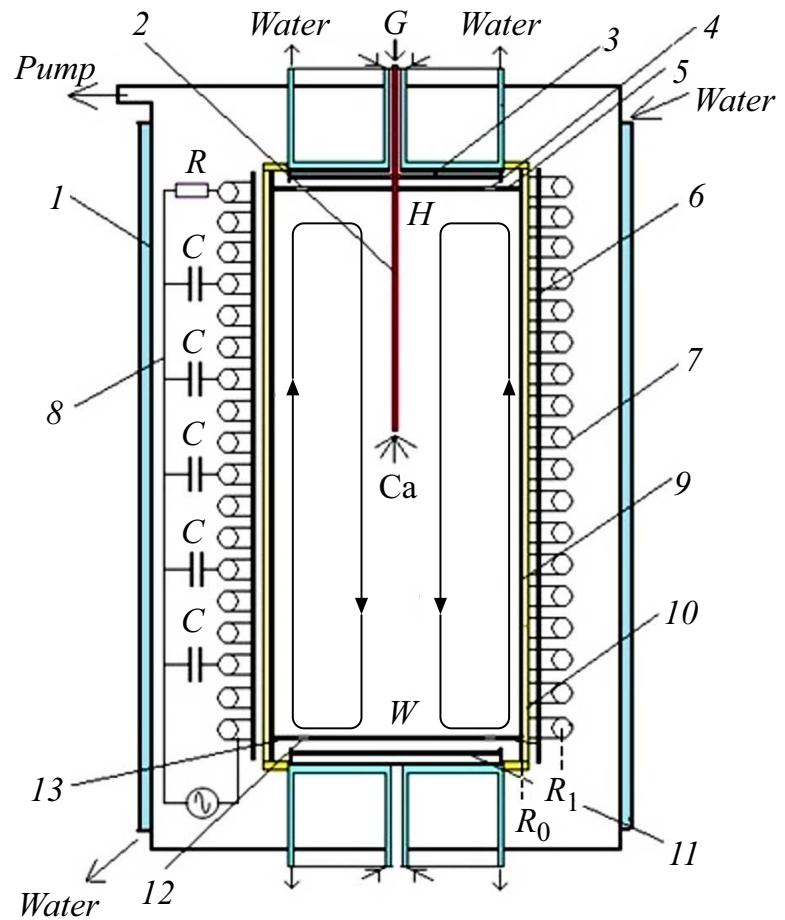

Рис. 1. Схема плазменной центрифуги с вращающимся магнитным полем и осевой циркуляцией, вызываемой бегущим магнитным полем. 1 - основная вакуумная камера; 2 - трубка для питания центрифуги; 3,11 - водоохлаждаемые диски для отбора тяжелого и легкого компонента; 4, 12 - отверстия, через которые отбирается обогащенный и обедненный продукт; 5,13 - горячие торцевые диски; 6 - токонесущие стержни для создания вращающегося магнитного поля; $7-$ спиральная обмотка для создания бегущего магнитного поля; 8 - линия задержки; 9 - металлическая стенка камеры разделения; 10 - цилиндрический слой жаропрочной керамики. $R_{0}-$ радиус стенки камеры разделения; $R_{1}-$ радиус обмотки бегущего поля; $G, H, W$ - потоки питания, отбора и отвала соответственно.

ках 5 и 13 соответственно. В процессе радиального разделения и одновременного продольного переноса компонентов на противоположных концах устройства концентрируются тяжелые (верхняя приторцевая зона) и легкие (нижняя зона) изотопы. При этом происходит непрерывный отбор через отверстия в горячих торцах и осаждение продукта на поверхностях отборных дисков.

Вращающееся магнитное поле создается системой токонесущих стержней 6 , два из которых показаны на передней проекции. Бегущее магнитное поле возбуждается спиральной обмоткой 7 с линией задержки 8 . Предположим, что бегущая магнитная волна распространяется в разделительной камере от нижнего торца к верхнему. Электромагнитная сила, вызванная действием бегущей волны, больше вблизи стенки, чем на оси. Поскольку в результате действия центробежной силы тяжелые изотопы отбрасываются к периферии, циркуляция способствует переносу тяжелого изотопа в верхнюю часть камеры.
Цилиндрическая стенка разделительной камеры, непосредственно соприкасающаяся с плазмой, должна быть диэлектриком, стойким к воздействию паров кальция. Вероятным кандидатом на эту роль могут быть высокотемпературные керамики типа $\mathrm{Al}_{2} \mathrm{O}_{3}$, температура которой должна поддерживаться в области $T_{\text {hot }} \approx 1300 \mathrm{~K}$, чтобы обеспечить необходимую плотность паров кальция. При выборе этого материала могут возникнуть трудности, связанные с неблагоприятными химическими и механическими свойствами керамик. В качестве альтернативного варианта можно изготавливать внутреннюю цилиндрическую стенку 9 из тонкого листа ниобия или подходящего сплава с высоким удельным сопротивлением. Однако при этом следует обеспечить проникновение высокочастотного поля в плазму. Вполне вероятно, что для осуществления пробоя понадобится дополнительный импульсный источник электрической энергии (например, искровой генератор).

Введем продольный коэффициент обогащения циркуляционной центрифуги с умножением первичного эффекта по высоте устройства $\varepsilon_{z}$, приходящийся на единичную разность масс разделяемых изотопов:

$$
\varepsilon_{z i}=\frac{c_{i p}-c_{i w}}{c_{i 0}\left(\langle\mu\rangle-\mu_{i}\right)},
$$

где величины $c_{i p}$ и $c_{i w}$ - локальные концентрации компонентов в отборе и отвале, $\mu_{i}$ - молярная масса компонента, $\langle\mu\rangle-$ средняя молярная масса. Величина $\varepsilon_{z}$ представляет собой только характеристику силового поля и не зависит от того, является ли смесь двухкомпонентной или многокомпонентной.

Уравнение переноса в двухкомпонентной смеси с единичной разностью масс для безотборного режима запишем в форме [1]:

$$
\left(K_{1}+K_{2}\right) \frac{d C}{d z}=K_{3} C(1-C),
$$

C - осредненная по радиусу мольно-долевая концентрация тяжелого компонента, $K_{1}, K_{2}$ и $K_{3}-$ коэффициенты, определяемые соотношениями

$$
\begin{gathered}
K_{1}=2 \pi \int_{0}^{R_{0}} \frac{\psi^{2}(r)}{\rho D r} d r, \quad K_{2}=2 \pi \int_{0}^{R_{0}} \rho D r d r, \\
K_{3}=2 \pi \int_{0}^{R_{0}} \psi(r) \varepsilon_{r}(r) d r,
\end{gathered}
$$

где $\psi(r)=\int_{0}^{r} \rho V_{z}(r) r d r-$ функция тока, $\rho-$ плотность смеси, $D-$ коэффициент радиальной диффузии нейтралов, $V_{z}(r)$ - осевая скорость плазмы, $\varepsilon_{r}(r)=V_{\varphi}^{2}(r) / \mathfrak{R} T_{n} r, T_{n}-$ температура нейтрального газа. Величина $V_{\varphi}(r)$ в режиме умеренных индуцированных магнитных полей [8] и в пренебрежении торможением среды в экмановских слоях на торцах камеры 


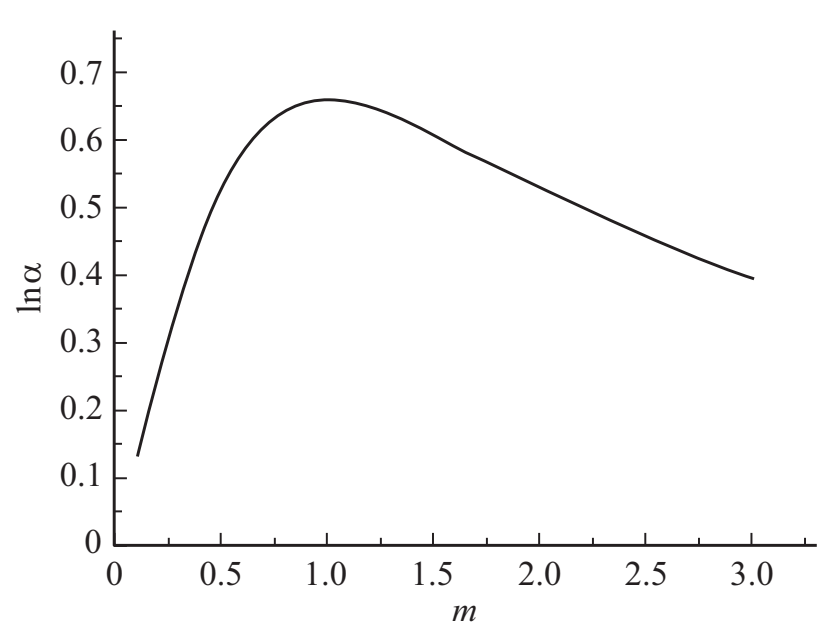

Рис. 2. Зависимость степени разделения плазменной центрифуги от параметра циркуляции $m$.

разделения [9] определяется выражением

$$
V_{\varphi}=\frac{B_{0}^{2} R_{0} \operatorname{Re}_{m}}{16 \mu_{0}\langle\eta\rangle \chi} y\left(1-y^{2}\right),
$$

где $B_{0}=I_{z 0} \mu_{0} / 2$ - амплитудное значение индукции вакуумного вращающегося магнитного поля, $I_{z 0}-$ амплитудное значение линейной плотности поверхностного осевого тока, возбуждающего вращающееся магнитное поле, $y=r / R_{0},\langle\eta\rangle-$ среднее значение коэффициента динамической вязкости, $\chi=\operatorname{ber}_{0}^{2}\left(\sqrt{\mathrm{Re}_{m}}\right)+$ $+b e i_{0}^{2}\left(\sqrt{\mathrm{Re}_{m}}\right)$ - безразмерный параметр, зависящий от магнитного числа Рейнольдса $\operatorname{Re}_{m}=\mu_{0} \sigma \omega_{0} R_{0}^{2}$ [8], $\operatorname{ber}_{0,1}$ и bei $_{0,1}$ - модифицированные функции Бесселя, $\sigma-$ проводимость плазмы, $\omega_{0}-$ частота вращения магнитного поля. Полагая давление нейтральных паров $P=15$ Torr, вкладываемую в плазму на единицу высоты устройства мощность $W=5 \mathrm{~kW} / \mathrm{m}$ $\left(\sigma=5 \cdot 10^{3} \mathrm{~S} / \mathrm{m}\right), \omega_{0}=2 \cdot 10^{5} \mathrm{~s}^{-1} \quad\left(\operatorname{Re}_{m} \approx 3, \quad \chi \approx 1.5\right)$, $\langle\eta\rangle=2.3 \cdot 10^{-5} \mathrm{~kg} /(\mathrm{m} \cdot \mathrm{s})$, получим для вакуумного поля величину $B_{0}=2.7 \cdot 10^{-3} \mathrm{~T}$, а для скорости вращения плазмы на радиусе $y=0.57$ значение $V_{\varphi} \approx 600 \mathrm{~m} / \mathrm{s}$.

Интегрируя (2) с учетом (3), найдем для коэффициента разделения следующее выражение:

$$
\begin{aligned}
\alpha & =(C /(1-C))_{z=L} /(C /(1-C))_{z=0} \\
& =\exp \left(\varepsilon_{1} \frac{L_{H}}{R_{0}} \frac{2 m}{1+m^{2}} N\right),
\end{aligned}
$$

где $L_{H}$ - длина отборной части колонны, $m=F / F_{0}$ безразмерный удвоенный циркуляционный поток,

$$
\begin{gathered}
F=2 \pi \int_{0}^{R_{0}}\left|\rho V_{z}(r)\right| r d r, \quad F_{0}=\sqrt{\frac{K_{2}}{k_{1}}}, \quad k_{1}=\frac{K_{1}}{F}, \\
N=\sqrt{2} \int_{0}^{1} y\left(1-y^{2}\right)^{2} \psi_{0} d y / \sqrt{\int_{0}^{1} \frac{\psi_{0}^{2}}{y} d y,}
\end{gathered}
$$

$$
\begin{gathered}
\psi_{0}=\frac{\psi}{V_{z 0} \rho_{0} R_{0}^{2}}=\exp (-A y)\left[\lambda\left(y^{2}-1\right)+1-y^{4}\right], \\
V_{z 0}=\frac{\sigma B_{1}^{2} R_{0}^{4} k_{z} \omega_{1} \operatorname{Re}_{m 1}}{128\langle\eta\rangle \chi_{1}}, \quad A=\frac{\langle\mu\rangle B_{0}^{4} R_{0}^{2} \operatorname{Re}_{m}^{2}}{3 \cdot 512 \Re\left(\mu_{0}^{2}\langle\eta\rangle^{2} \chi^{2}\right.}, \\
\varepsilon_{1}=\frac{B_{0}^{4} R_{0}^{2} \operatorname{Re}_{m}^{2}}{256\langle\eta\rangle^{2} \mu_{0}^{2} \Re T_{0} \chi^{2}}, \quad k_{z}=\frac{\omega_{1}}{V_{p h}},
\end{gathered}
$$

$\operatorname{Re}_{m 1}=\mu_{0} \sigma \omega_{1} R_{0}^{2}, \lambda$ и $\chi_{1}=\left(f_{1}^{2}+f_{2}^{2}\right)$ - безразмерные коэффициенты, определенные в [8].

Для достижения оптимальной циркуляции при значениях параметров $y_{1}=R_{1} / R_{0}=1.2, \quad V_{p h}=10^{3} \mathrm{~m} / \mathrm{s}$, $\chi_{1}=f_{1}^{2}+f_{2}^{2} \approx 270$ амплитудное значение индукции бегущего магнитного поля должно быть равно $B_{1}^{*} \approx 4 \cdot 10^{-3} \mathrm{~T}$.

На рис. 2 приведена зависимость логарифма коэффициента разделения отборной части $\ln \alpha$ от параметра, характеризующего интенсивность осевой циркуляции $m$, при температуре $T_{n}=1300 \mathrm{~K}$. Учитывая, что $\ln \alpha \approx \varepsilon_{z i}$, с помощью уравнения (1) легко оценить продольные градиенты концентрации для всех компонентов разделяемой многокомпонентной смеси. Отметим, что рассчитанные в работе максимальные степени разделения $\left(\ln \alpha_{m} \approx 0.7\right)$ превышают эффекты, достигаемые в разделительных колоннах, используемых в диффузионных методах разделения изотопов.

Авторы благодарны Дж. Вичелло (J. Whichello), Департамент прикладной и плазменной физики Университета Сиднея (Австралия), за полезное обсуждение работы.

Работа поддержана Российским фондом науки (грант 18-19-00447).

\section{Список литературы}

[1] Борисевич В.Д., Борман В.Д., Сулаберидзе Г.А., Тихомирова А.В., Токманцев В.И. Физические основы разделения изотопов в газовой центрифуге. Ч. II. Разделение многокомпонентных изотопных смесей. М.: МЭИ. 2011. 275 с.

[2] Fetterman A.J., Fisch N.J. // Phys. Plasmas. 2011. V. 18. P. 094503.

[3] Fetterman A.J., Fisch N.J. // Plasma Sources Sci. Technol. 2009. V. 18. P. 045003.

[4] Гориунов Н.М., Горкунов А.А., Горкунов М.А., Потанин Е.П. // ПТЭ. 2015. Т. 58. № 6. С. 45-50.

[5] Oganessian Yu.Ts., Abdullin F.Sh., Bailey P.D., Abdullin F.Sh., Bailey P.D., Benker D.E., Bennett M.E., Dmitriev S.N., Ezold J.G., Hamilton J.H., Henderson R.A., Itkis M.G., Lobanov Yu.V., Mezentsev A.N., Moody K.J., Nelson S.L., Polyakov A.N., Porter C.E., Ramayya A.V., Riley F.D., Roberto J.B., Ryabinin M.A., Rykaczewski K.P., Sagaidak R.N., Shaughnessy D.A., Shirokovsky I.V., Stoyer M.A., Subbotin V.G., Sudowe R., Sukhov A.M., Tsyganov Yu.S., Utyonkov V.K., Voinov A.A., Vostokin G.K., Wilk P.A. // Phys. Rev. Lett. 2010. V. 104. P. 142502.

[6] Bonvicini V., Capelli S., Cremonesi O., Cucciati G., Gironi L., Pavan M., Previtali E., Sisti M. // Eur. Phys. J. C. 2014. V. 74. P. 3151. arXiv:1407.4608. 
[7] Niki H., Teranishi K., Fujita T., Hashizume K., Ezaki Y., Ogawa I. Laser isotope separation of calcium: deflection of $\mathrm{Ca}$ atomic beam by a diode laser // Proc. 14th Int. Workshop on separation phenomena in liquids and gases. Stresa, Italy, 2017. P. 108-112.

[8] Borisevich V.D., Potanin E.P. // Phys. Scripta. 2017. V. 92. P. 075601.

[9] Borisevich V.D., Potanin E.P., Whichello J. // J. Fluid Mech. 2017. V. 829. P. 328-244. 